\title{
Extracellular matrix expansion by cardiac magnetic resonance T1 mapping- validation with myocardial biopsy
}

\author{
Andreas Kammerlander ${ }^{1 *}$, Caroline Tufaro ${ }^{1}$, Alina F Bachmann ${ }^{1}$, Beatrice A Marzluf ${ }^{1}$, Stefan Aschauer ${ }^{1}$, \\ Andreas Greiser², Diana Bonderman', Julia Mascherbauer' \\ From 18th Annual SCMR Scientific Sessions \\ Nice, France. 4-7 February 2015
}

\section{Background}

Accumulation of diffuse myocardial fibrosis / extracellular volume (ECV) in the myocardium is common to various cardiac diseases and is associated with an unfavorable prognosis. Various cardiac magnetic resonance (CMR) T1 mapping techniques have recently been proposed for the quantification of ECV, including:

1. Modified Look-Locker Inversion recovery (MOLLI) T1 mapping including calculation of ECV, 2. Post-contrast multiple-breath-hold T1 mapping. 3. Native (precontrast) T1 mapping.

T1 mapping is a promising technique, however, validation data based on myocardial biopsy are sparse.

\section{Methods}

36 heart failure patients underwent CMR T1 mapping and left-ventricular biopsy within 4 weeks. CMR protocols (1.5-T scanner, (Magnetom Avanto, Siemens Healthcare, Erlangen, Germany) included non-product MOLLI and multiple-breath-hold T1 mapping.

The population consisted of 22 patients with HFpEF (heart failure with preserved ejection fraction), 7 with cardiac amyloidosis, 3 with HFrEF (heart failure with reduced ejection fraction) and 4 with organic mitral regurgitation (MR).

Specimens were stained using modified Trichrome. TissueFAXS and dedicated software were used to quantify ECV.

\section{Results}

ECV by TissueFAXS was $32 \pm 17 \%$ and $35 \pm 13 \%$ by CMR MOLLI. Post-contrast T1 times by the multiple-breathhold sequence were $411 \pm 79 \mathrm{~ms}$, native $\mathrm{T} 1$ times were $1009 \pm 73 \mathrm{~ms}$. Patients with amyloidosis had significantly more ECV by TissueFAXS $(59 \pm 13 \%, \mathrm{p}<0.001)$ and with CMR MOLLI $(56 \pm 12 \%, \mathrm{p}<0.001)$ compared with the other pathologies.

The amount of TissueFAXS ECV correlated significantly with native $\mathrm{T} 1$ times $(\mathrm{r}=0.657, \mathrm{p}<0.001)$, MOLLI ECV ( $r=0.907, p<0.001)$ and with multiple-breath-hold post-contrast T1 times $(\mathrm{r}=-0.683, \mathrm{p}<0.001)$. When excluding patients with amyloidosis, only MOLLI ECV significantly correlated with histology $(r=0.491$, $\mathrm{p}=0.015)$, whereas multiple-breath-hold post-contrast $\mathrm{T} 1$ times just missed the level of significance $(r=-0.413$, $\mathrm{p}=0.056)$.

\section{Conclusions}

In our series, MOLLI ECV appears to be the most promising method for the quantification of extracellular matrix when validated against histology. Future studies need to define the prognostic value of T1 mapping in a large prospective cohort.

\section{Funding}

The study received support from the Österreichischer Herzfonds and the Austrian fellowship grants KLI 245.

\footnotetext{
Authors' details

${ }^{1}$ Medical University of Vienna, Vienna, Austria. ${ }^{2}$ Siemens AG Healthcare Sector, Erlangen, Germany.
} 
- Convenient online submission

- Thorough peer review

- No space constraints or color figure charges

- Immediate publication on acceptance

- Inclusion in PubMed, CAS, Scopus and Google Scholar

- Research which is freely available for redistribution 